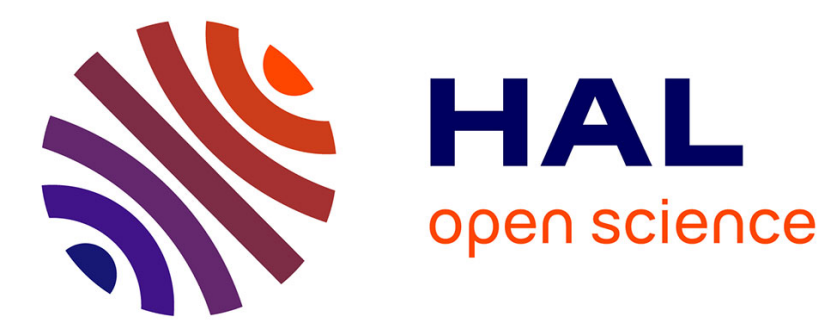

\title{
Potential Field based Approach for Coordinate Exploration with a Multi-Robot Team
}

Alessandro Renzaglia, Agostino Martinelli

\section{To cite this version:}

Alessandro Renzaglia, Agostino Martinelli. Potential Field based Approach for Coordinate Exploration with a Multi-Robot Team. 8th IEEE International Workshop on Safety, Security and Rescue Robotics (SSRR), Jul 2010, Bremen, Germany. inria-00525789

\section{HAL Id: inria-00525789 \\ https://hal.inria.fr/inria-00525789}

Submitted on 12 Oct 2010

HAL is a multi-disciplinary open access archive for the deposit and dissemination of scientific research documents, whether they are published or not. The documents may come from teaching and research institutions in France or abroad, or from public or private research centers.
L'archive ouverte pluridisciplinaire HAL, est destinée au dépôt et à la diffusion de documents scientifiques de niveau recherche, publiés ou non, émanant des établissements d'enseignement et de recherche français ou étrangers, des laboratoires publics ou privés. 


\title{
Potential Field based Approach for Coordinate Exploration with a Multi-Robot Team
}

\author{
Alessandro Renzaglia and Agostino Martinelli \\ INRIA Rhône-Alpes \\ Grenoble, France \\ Email: \{alessandro.renzaglia|agostino.martinelli\}@inrialpes.fr
}

\begin{abstract}
In this paper we introduce a new distributed algorithm for the exploration of an unknown environment with a team of mobile robots. The objective is to explore the whole environment as fastest as possible. The proposed approach is based on the potential field method. The advantages of using this method are several and well known, but the presence of many local minima does not assure the exploration of the entire environment. Our idea is to preserve these advantages but overcome the problem of local minima by introducing a leader in the team which has a different control law, unaffected by this problem. Furthermore, we consider also the case of several local leaders, dynamically selected on the basis of a hierarchy within the team. Extensive simulations are presented to evaluate the performance of the algorithm. In particular, the results are compared with the exploration obtained by a potential field approach without leaders.
\end{abstract}

Keywords: multi-robot system, cooperative exploration, autonomous agents.

\section{INTRODUCTION}

Exploring unknown environments is a task of fundamental importance in mobile robotics. Its applications are all of great relevance: mapping, rescue mission, searching, etc. Using coordinate algorithms for multi-robot teams allows us to achieve the task in reduced time and increases the probability of success. Another advantage is that multiple robots may produce more accurate maps, due to merging of overlapping information. This can help compensate for sensor uncertainty and localization error, especially when the robots have different sensor and/or localization capabilities [6]. For these reasons, in recent years, multi-robot exploration strategies have attracted increased attention from the research community. On the other hand, when many small robots are deployed to cooperatively explore an environment, several new problems arise due to the limited communication and computational capabilities of each agent. In particular, an algorithm whose computational cost is too heavy cannot be implemented. Furthermore, due to the limited communication capabilities, a suitable algorithm must only require communication among agents which are close. Finally, due to the possibility of communication failures, a centralized algorithm cannot be considered. An efficient solution must be able not only to distribute the computation but also to dynamically adapt in order to face any communication failure.

In this paper we consider the problem of exploring a planar environment with unknown obstacles by means of a team of mobile robots. Thus, the goal is minimizing the time required to cover the whole region to get a map of the environment. The robot team is assumed able to sharing information to get a common map of the explored environment. The main problem to solve is to find for each time step the optimal next target point for each robot of the team.

The exploration strategy here adopted is based on the concept of frontier cells, introduced for the first time in [1]. In [2] the authors propose a frontier based probabilistic strategy for the exploration by using a single mobile robot. Burgard et al. have developed a frontier based algorithm to coordinate a multi-robot team, which simultaneously takes into account the cost and the utility of reaching a point of the frontier [3], [4]. In [5] the author proposes a strategy based only on the artificial forces: each robot feels the repulsion of the other robots, of the obstacles and the attraction of the closest point of the frontier. For many environments this approach works very well. The drawback, as in every potential field based approach, is the presence of local minima. Because of this problem, it is possible that the robot team is not able to explore the whole environment and this is the most important limitation of this method. In [7] the authors present a communicative exploration algorithm with the purpose of exploring an unknown environment taking into account the constraints of wireless networking. The frontier based approach is not the only one so far proposed, for example in [8] the authors used a random selection mechanism.

A different kind of exploration problems present in literature is the cleaning like problem. In this case the aim is visiting each location in a known terrain. The strategies for these problems are usually based on subdividing the work-area into disjoint cells [9], [10]. A survey of results for this kind of coverage problem can be found in [11], where the author classifies the existing approaches in: heuristic and randomize, approximate cellular decomposition based and exact cellular decomposition based.

The use of a potential field approach to coordinate a multi robot system is present in literature for many other different tasks. In particular, it has been applied for tasks in which is useful getting a good spreading out of the team. For example, in the field of safety and security robotics, this method has been used for cooperative surveillance problem in complex environments, where an analytical solution is impossible to find [16], [17]. In [18] the authors use the same approach to 
obtain a coverage of a convex region with the constraint that each robot has at least $K$ neighbors.

In this work we propose a new coordinate and distributed algorithm for the exploration of an unknown nonconvex environment by using a team of mobile robots. Our intent is to exploit the well known advantages of the potential field method to achieve the task. Indeed, this method, introduced for the first time by Khatib [12], is an elegant, distributed and fast mean to get collision-free trajectories (e.g. see [15]). Furthermore, the repulsion between the robots ensures also a useful spreading out of the team. However, it is very easy that local minima cause the stopping of the mission and then the completely exploration is not assured. By introducing a leader in the team whose control is unaffected by local minima, we can eliminate the problem of robots' stalling. Then, we consider also the case in which a single leader is not enough to have a good solution. Problems of communication, operation or a large spreading out of the team during the task may be reasons to introduce several local leaders.

This paper is organized as follows. In the next section we present the formulation of the cooperative exploration problem. In section III we explain in details the proposed method describing the different control law for the leader and for the other robots. Then, we extend the proposed solution also for the case in which it is necessary the presence of several local leaders within the team. In section IV several numerical simulations with different initial conditions and different environments are made and compared, to evaluate the performance of our algorithm. In particular, we show the difference of a potential field based approach for exploration with and without the presence of local leaders.

\section{PROBLEM FORMULATION}

Let us consider a planar environment with unknown obstacles and a team of mobile robots. We suppose that each robot has an omnidirectional range sensor, with a limited radius of detection, able to measure the distances robot-obstacle and robot-robot. A point in the environment is considered explored when at least one of the team's members approaches this point by a distance smaller than the radius of detection of its sensor. The goal we want to achieve is to explore the whole environment as fastest as possible. Thus, it is necessary to coordinate the robots to avoiding as most as possible the overlapping of information and, at the same time, ensure the success of the exploration. A solution of this problem consists in the assignment of the target cell for every robot at every time step.

To achieve the task we assume also that each robot is able to share a common grid based map of the explored region. In particular, each cell in the grid is classified in three different ways:

- Explored: cell containing only points which have been explored;

- Unexplored: cell containing only points which have not been explored;

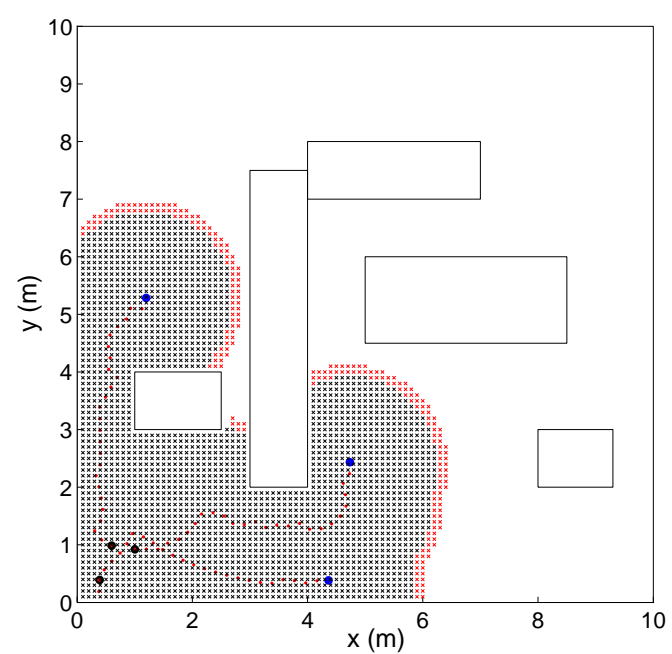

Fig. 1. Exploration in progress. The environment is divided in explored areas (in black), unexplored (in white) and the frontier (in red). For the robots' motion: the black points show the positions of the robots at the beginning of the mission, the final ones are in blue, in red the trajectories.

- Frontier: cell containing both explored and not explored points.

For the single robot case, a convenient choice for the best next target is the closest cell, taking into account the topology of the environment, within the set of the frontier cells (see [1]). In fig. 1 it is shown a typical configuration during an exploration mission: the environment is divided in explored areas, unexplored areas and frontier.

For the multi-robot problem, to choose which cell of the frontier is the target for one robot we should consider both the cost and the utility of it. This is fundamental because the same cell of the frontier could be the optimal cost next target for more than one robot. To solve this problem we consider an algorithm based on the artificial potential field method which combines the attraction toward the closest frontier cell and a repulsion between the robots. The repulsion between the robots is an efficient and easy mean to avoid that two or more robots choose the same target cell. Considering also a repulsion from the obstacles allows getting collision-free trajectories. However, to be sure that local minima does not forbid the task accomplishment, one robot of the team, the leader, is subject to a different control law. It always reaches the closest cell of the frontier by traveling along the shortest collision-free path, regardless what the other robots do.

\section{PROPOSED METHOD}

In this section we explain with more details the proposed method. In particular, we describe the two different control laws for the leader and for the other robots of the team. Then, we explain how extend the idea for a dynamical selection of several local leaders based on a hierarchy within the team. 


\section{A. Leader}

As already mentioned, the reason to introduce a leader is to avoiding that the presence of local minima stops the exploration before having accomplished the task. Thus, its control law must be unaffected by local minima. To satisfy this condition the leader has a completely autonomous motion which does not depend on the other robots. It gets its closest point belonging to the frontier by following the shortest collision-free path, i.e. avoiding the obstacles. In particular, to find it, we have considered the visibility graph of the already detected area and identified the optimal path by using the Dijkstra's algorithm [13], [14]. Obviously, this is only one of the possible methods to find the optimal path, but a too "heavy" algorithm is not within the philosophy of this work.

\section{B. Trooper}

The motion of the other robots is generated by an artificial potential field composed by the sum of:

- a repulsion from the other robots (including the leader);

- a repulsion from the closest detected obstacle;

- an attraction toward the closest cell of the frontier.

1) Repulsive potential: The repulsive potential used in the model is:

$$
U_{r e p}\left(\mathbf{q}, \mathbf{q}_{i}\right)= \begin{cases}\frac{1}{2} k_{r e p}\left(\frac{1}{\rho(\mathbf{q})}-\frac{1}{\rho_{0}}\right)^{2} & , \rho(\mathbf{q}) \leq \rho_{0} \\ 0 & , \rho(\mathbf{q})>\rho_{0}\end{cases}
$$

where $\mathbf{q}_{i}$ is the position of the robot/obstacle, $\rho(\mathbf{q})=\| \mathbf{q}-$ $\mathbf{q}_{i} \|$ and $\rho_{0}$ is the range of the interaction. The artificial force induced by this potential field is $\mathbf{F}(\mathbf{q})=-\nabla U(\mathbf{q})$ :

$$
\mathbf{F}_{r e p}\left(\mathbf{q}, \mathbf{q}_{i}\right)= \begin{cases}k_{r e p}\left(\frac{1}{\rho(\mathbf{q})}-\frac{1}{\rho_{0}}\right) \frac{\mathbf{q}-\mathbf{q}_{i}}{\rho^{3}(\mathbf{q})} & , \rho(\mathbf{q}) \leq \rho_{0} \\ 0 & , \rho(\mathbf{q})>\rho_{0}\end{cases}
$$

Then, each robot feels a total repulsive force equals to:

$$
\mathbf{F}_{r e p}(\mathbf{q})=\sum_{i=1}^{N} \mathbf{F}_{r e p}\left(\mathbf{q}, \mathbf{q}_{i}\right)
$$

where the sum is over the other $N-1$ robots and the closest obstacle. In general, the range of interaction $\rho_{0}$ for the obstacles repulsion and for the robots repulsion has to be different. In particular we have

$$
\rho_{0}^{o b s} \ll \rho_{0}^{r o b} .
$$

This condition is necessary because the repulsion from the obstacle is introduced only to avoid collision, whilst the repulsion between the robot has also the intent to well spread out the team during the exploration.

2) Attractive potential: The attractive potential employed is:

$$
U_{\text {att }}(\mathbf{q})=\frac{1}{4} k_{\text {att }} \rho_{\text {goal }}^{4}
$$

and the corresponding force

$$
\mathbf{F}_{a t t}(\mathbf{q})=k_{a t t}\left(\mathbf{q}_{\text {goal }}-\mathbf{q}\right) \rho_{\text {goal }}^{2}
$$

where $\rho_{\text {goal }}=\left\|\mathbf{q}-\mathbf{q}_{\text {goal }}\right\|$ and $\mathbf{q}_{\text {goal }}$ is the position of the closest point of the frontier. Added to these forces, we have also considered a viscous term, $\nu v$, in order to get more regular trajectories. The equation of motion is:

$$
\mathbf{F}_{t o t}=\mathbf{F}_{r e p}+\mathbf{F}_{a t t}=m \ddot{\mathbf{q}}-\nu \dot{\mathbf{q}}
$$

where $m$ is the virtual robot mass which, without any loss of generality, we assume unitary.

\section{Several Leaders}

In many cases only one leader might be not enough. First of all, we always have to consider possible failures of the leader during the mission. Problems of communication are always possible. Furthermore, a very probably scenario might be that the collaborative exploration drives to the spreading out of the team. When this effect becomes very strong, in relation to the radius of detection of the robots' sensors, we may lose the collaborative nature of the team. In this case may be useful consider more sub-teams, each one with its leader. In other words, the leadership must be dynamic during the mission. Our solution is the following: when a robot does not detect any leader within a circle of fixed radius $r_{l e a d}$, it becomes a leader. Contrary, when a local leader detects again an other leader, one of them lose its leadership. To decide who remains leader, it is necessary fixing at the beginning a hierarchy between the robots. With respect to the range of interaction for the robot repulsion previously introduced, $\rho_{0}^{r o b}$, is always verified the relation

$$
\rho_{0}^{r o b} \ll r_{\text {lead }} .
$$

In this way, each sub-team has both the cooperative behaviour, given by the potential field approach of the nonleader robots, and the certainty of continuing exploration until a frontier is present.

Once introduced the sub-teams, we can also discuss the requests on the communication capabilities of the robots. Obviously, a complete communication between all the robots might be useful: in this case each robot has a complete map of the whole explored region and so, of the whole existing frontier. However, because of the distributed properties of the algorithm this is not necessary. Indeed, the only important request is that there is a good communication within each sub-team. In terms of range of communication $R_{\text {com }}$, we can express such condition in the following way:

$$
R_{\text {com }} \geq r_{\text {lead }} .
$$

In this way it is assured that each local leader can receive all the data stored by the robots of its sub-team and so, that the exploration task will be completed. On the other hand, the previous condition can be also used to fix the parameter $r_{\text {lead }}$ knowing the communication capabilities of the robots, i.e. $R_{\text {com }}$.

Finally, it is also important to emphasize that for a well behaviour of a multi-robot team with a potential field based control it is of crucial importance a right choice of the potentials' parameters. These parameters strongly depend on 


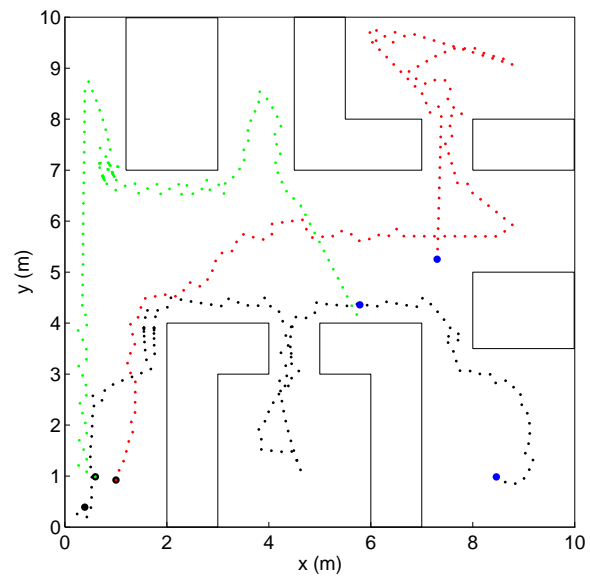

Fig. 2. Exploration of a nonconvex environment by a team of three homogeneous robots. The initial positions are in black the finale ones in blue. The trajectories are in different colors for each robot.

the particular kind of the environment (form of the obstacles, narrowness of the passages, etc): thus, they depend on several properties which are unknown at the beginning of the exploration. This is the most important problem of using this method and the cause of the presence of many stalling points. So, the possibilities that more robots can become leaders and thus coming out from stalling positions is relevant in practical applications.

\section{Numerical Simulations}

In this section we show several numerical simulations to evaluate the performance of our algorithm. In particular, we compare the proposed method with the potential field based approach without leaders. In the simulations we did not introduce any assumption on the topology of the obstacles. On the other hand, the external boundary of the environment in the proposed simulations is always convex. This choice has been made to emphasize that also in simple environment the presence of leaders is of crucial importance in a potential field approach. At the beginning of the simulation only one robot is leader and it remains leader throughout the task. For the other robots, a hierarchy is initially defined and they can become temporary leaders as explained in the previous section. In the simulation we have also considered cinematical constraints for the robots' motion. In particular, each robot has a maximum speed $\left|v_{\max }\right|=1 \mathrm{~m} / \mathrm{s}$.

The first simulation, in fig. 2, shows the application of the proposed method for the exploration of a complex environment by a team of three robots. It is clear the cooperative behaviour which brings to spread out the teammates in order to explore different areas of the environment.

In the other simulations we want to prove how the presence of a leader is of crucial importance in a potential field approach. In fig. 3 it is possible to see a typical situation where the presence of the leader allows achieving the exploration (fig. 3(a)) and, in the same time, the team without leaders

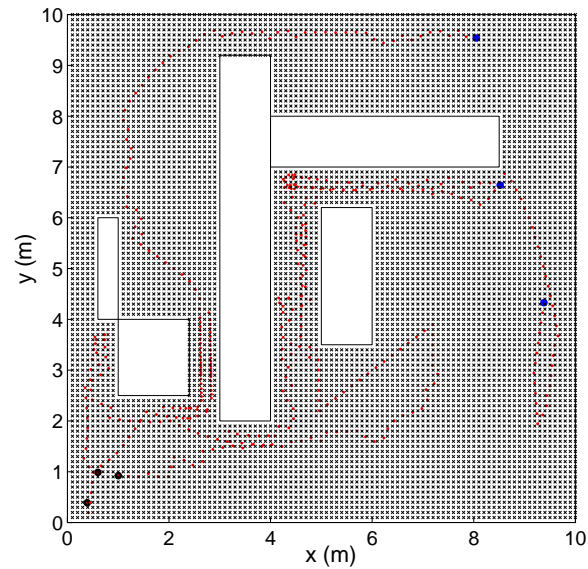

(a)

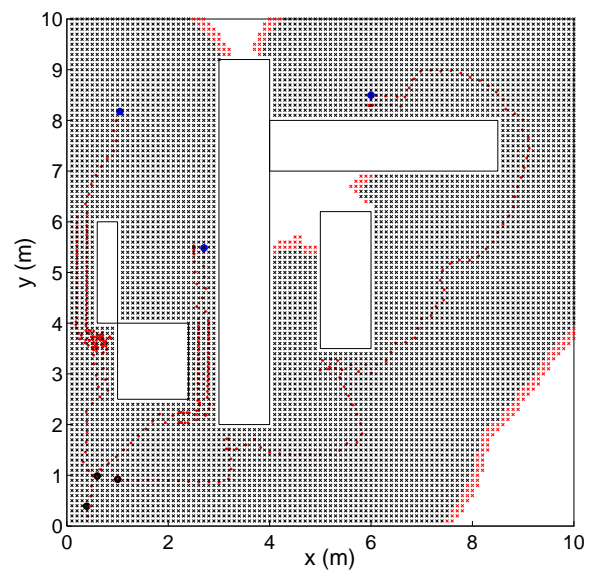

(b)

Fig. 3. The exploring team is composed by three homogeneous robots with a radius of detection of $2.5 \mathrm{~m}$. In fig. (a) the robot team explores the whole environment by using the proposed algorithm. After the same time, in fig. (b), without any leaders in the team, the exploration is not accomplished.

(fig. 3(b)) is not able to complete the task. In this case the three homogeneous robots of the team have omnidirectional range sensors with maximum distance of detection $r=2.5 \mathrm{~m}$.

The same situation, in the same environment but with a different robot team is shown in fig. 4. The team is now composed by four robots with a smaller maximum distance of detection $r=1.5 \mathrm{~m}$ and with a different starting deployment. In this case even without leaders in the team the exploration is achieved, but in a longer time. Indeed, as it is possible to see in fig. 5, where the percentage of explored space in function of time are shown, both the methods are able to complete the task. However, the proposed approach is faster because it has 


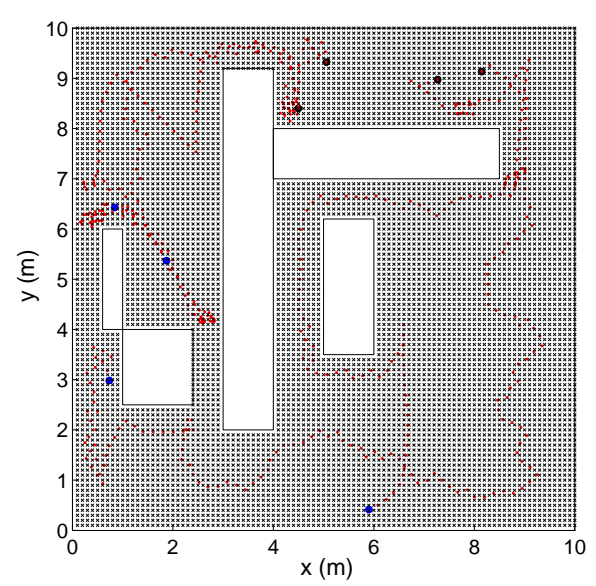

(a)

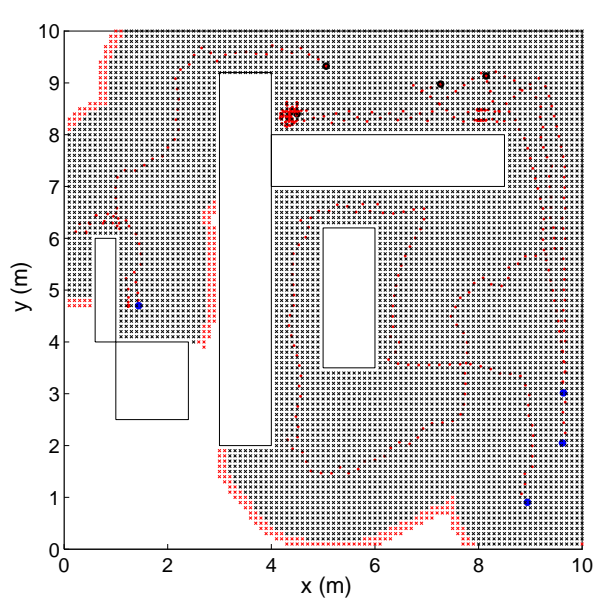

(b)

Fig. 4. Same scenario than in fig. 3. Now the exploring team is composed by four homogeneous robots with a radius of detection of $1.5 m$.

less problem of stalling for the robots.

The last proposed simulation (fig. 6) shows the same behaviour of the previous simulations. In this case the team is composed by three homogeneous robots with a maximum distance of detection $(r=2 m)$ and the environment to explore has different obstacles. However, in this case, the only potential field approach is not able to accomplish the task. As it is clear from the cost functions in fig. 7, without leaders the team reaches a local minimum and it cannot get over it. The result is that it cannot finish the exploration of the environment. On the other hand, the cost function obtained by the proposed method is a monotonically increasing function which does not present any stalling configuration.

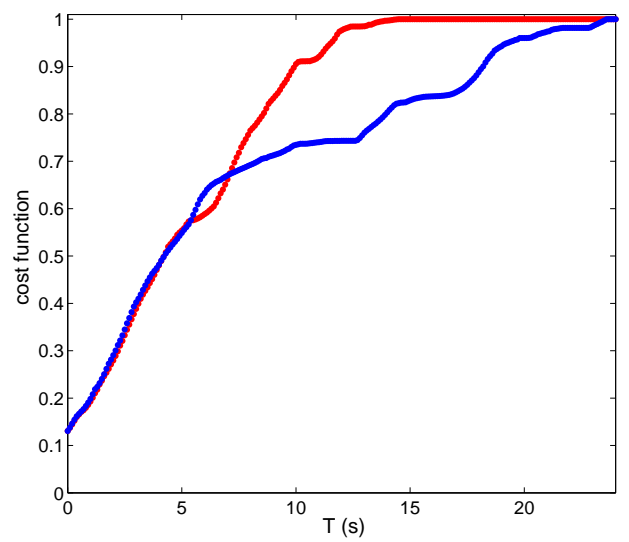

Fig. 5. Percentages of the space explored obtained by the two methods, for the simulations shown in fig. 4. In red is the results obtained by using the proposed method.

\section{CONCLUSION AND FUTURE WORKS}

We have presented a new coordinate and distributed algorithm for the exploration of a nonconvex unknown environments by using a team of mobile robots. The algorithm is based on the potential field method but with the inclusion of a leader in the team which allows avoiding the problem of local minima and assures the accomplishment of the task for every kind of environment. We have considered also cases in which communication problems or a too large spreading out of the team during the mission requires the presence of several sub-teams, each one with its leader. So the local leadership becomes a dynamic figure. Several experiment simulations prove that the proposed method allows achieving the exploration for different environments and different robot team conditions.

Future works will focus mainly on the extension to the $3 \mathrm{D}$ case. In particular, our aim is to develop a strategy for the cooperative exploration of an unknown urban-like environment with a MAV swarm. For this case we will consider a cone of visibility for each robot, instead of an omnidirectional vision. Thus, apart from the position the orientation of each robot has to be considered.

\section{ACKNOWLEDGMENTS}

The research leading to these results has received funding from the European Community's Seventh Framework Programme (FP7/2007-2013) under grant agreement n. 231855 (sFly).

\section{REFERENCES}

[1] B. Yamauchi, "Frontier-based exploration using multiple robots," in 2nd Int. Conf. Autonomous Agents, pp. 47-53, 1998.

[2] L. Freda and G. Oriolo, "Frontier-based probabilistic strategies for sensor-based exploration," in IEEE International Conference on Robotics and Automation, Barcelona, Spain, pp. 3892-3898, 2005.

[3] W. Burgard et al., "Collaborative multi-robot exploration," in IEEE International Conference on Robotics and Automation, San Francisco, CA, USA, pp. 476-481, 2000. 


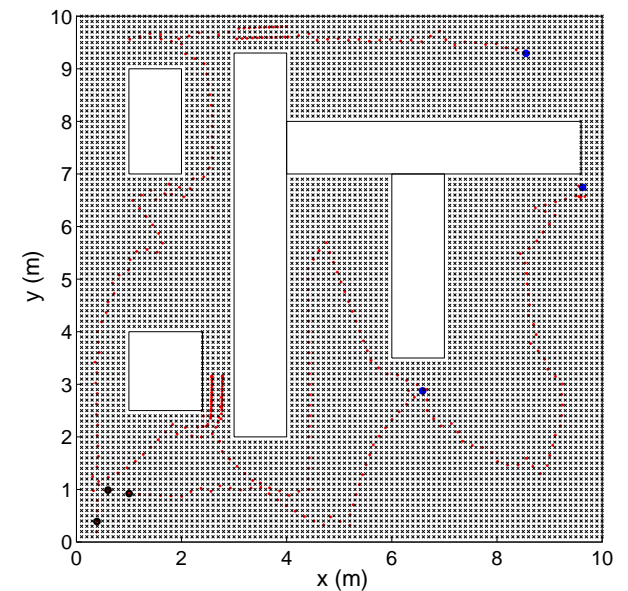

(a)

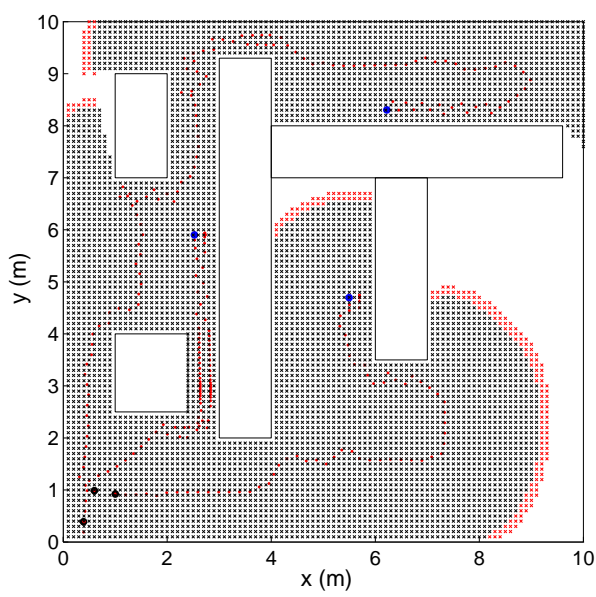

(b)

Fig. 6. Exploration of a different environment. The team is composed by three homogeneous robots with a radius of detection of $2 m$. Also in this case, without leaders, the accomplishment of the task is not achieved.

[4] W. Burgard, M. Moors, C. Stachniss and F.E. Schneider, "Coordinate multi-robot exploration," IEEE Transactions on Robotics, vol. 21 , no. 3 , pp. 376-386, 2005.

[5] H. Lau, "Behavioural approach for multi-robot exploration," in Australasian Conference on Robotics and Automation, Brisbane, Australia, 2003.

[6] D. Fox, W. Burgard, H. Kruppa and S. Thrun, "Collaborative multirobot localization," in Proc. 23rd German Conference on Artificial Intelligence, Springer-Verlag, 1999.

[7] M.N. Rooker and A. Birk, "Multi-robot exploration under the constraints of wireless networking," Control Engineering Practice, vol. 15, no. 4, pp. $435-445,2007$.

[8] S. Thrun, "Exploration in active learning," in The Handbook of Brain Theory and Neural Networks, MIT Press, pp. 381-384, 1998.

[9] Y. Gabrieli and E. Rimon, "Spanning-tree based coverage of continuous areas by a mobile robot,“ Ann. Math. Artif. Intell., vol. 31, pp. 7798,

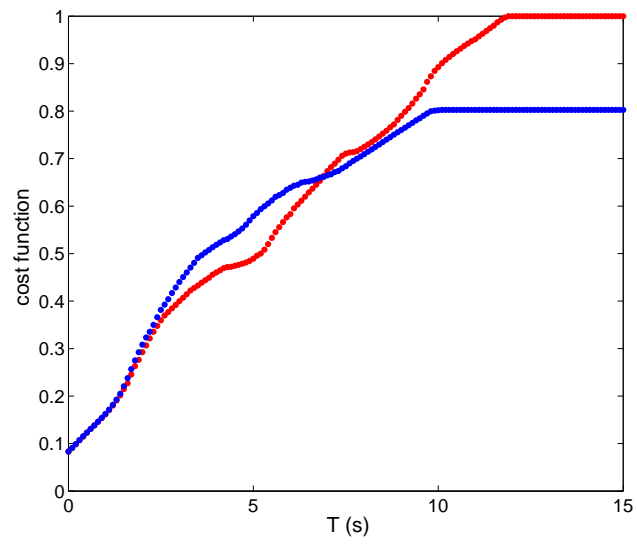

Fig. 7. Percentages of the space explored obtained by the two methods, for the simulations shown in fig. 6. In red is the results obtained by using the proposed method. The blue line show that without leaders the team is not able to explore the whole environment.

2001.

[10] X. Zheng, S. Jain, S. Koenig, D. Kempe, "Multi-robot forest coverage," IEEE/RSJ International Conference on Intelligent Robots and Systems, pp. 3852-3857, 2-6 Aug. 2005.

[11] H. Choset, "Coverage for robotics - A survey of recent results," Ann. Math. Artif. Intell., vol. 31, pp. 113-126, Jan. 2001.

[12] O. Khatib, "Real-time obstacle avoidance for manipulators and mobile robots," International Journal of Robotics Research, vol. 5, no. 1, pp. 90-98, 1986.

[13] T. Lozano-Perez and M.A. Wesley, "An algorithm for planning collisionfree paths among polyhedral obstacles," Commun. ACM, vol. 22, no. 10 , pp. 560-570, 1979

[14] E.W. Dijkstra, "A note on two problems in connexion with graphs," Numer. Math., vol. 1, no. 1, pp. 269-271, 1959.

[15] J.C. Latombe, "Robot Motion Planning, " Kluwer Academic Publishers, Boston, MA, 1991.

[16] A. Howard, M.J. Matarić and G.S. Sukhatme, "Mobile sensor network deployment using potential fields: A distributed, scalable solution to the area coverage problem," in 6th International Conference on Distributed Autonomous Robotic System (DASRSO2), Fukoka, Japan, pp. 299-308, 2002.

[17] A. Renzaglia and A. Martinelli, "Distributed Coverage Control for a Multi-Robot Team in a Non-Convex Environment,“ in IEEE IROSOS 3rd Workshop on Planning, Perception and Navigation for Intelligent Vehicles, St Louis, MO, USA, 2009.

[18] S. Poduri, G.S. Sukhatme, "Constrained Coverage for Mobile Sensor Networks," in IEEE International Conference on Robotics and Automation, New Orleans, LA, USA, pp. 165-172, 2004. 